\title{
Impact of Electric Vehicles on the Expansion Planning of Distribution Systems considering Renewable Energy, Storage and Charging Stations
}

\author{
Pilar Meneses de Quevedo, Gregorio Muñoz-Delgado, Student Member, IEEE and Javier Contreras, Fellow, IEEE
}

\begin{abstract}
Energy storage systems (ESS) have adopted a new role with the increasing penetration of electric vehicles (EVs) and renewable energy sources (RES). EVs introduce new charging demands that change the traditional demand profiles and RES are characterized by their high variability. This paper presents a new multistage distribution expansion planning model where investments in distribution network assets, RES, ESS and EV charging stations (EVCS) are jointly considered. The charging demand necessary for EVs transportation is performed using a vehicle model based on travel patterns. The variability associated with RES along with the demand requires the incorporation of uncertainty, which is characterized through a set of scenarios. These scenarios are generated by the k-means++ clustering technique that allows keeping the correlation in the information of the uncertainty sources. The resulting stochastic program is driven by the minimization of the present value of the total expected cost including investment, maintenance, production, losses and non-supplied energy. The associated scenario-based deterministic equivalent is formulated as a mixed-integer linear program, which can be solved by commercial software. Numerical results are presented for an illustrative 54-node test system.
\end{abstract}

Index Terms-Clustering technique, distribution system expansion planning (DSEP), ESS, EV charging demand, renewable energy.

$A$. Indexes
$a$
$b$
$c h$
$i, j$
$k$
$l$
$p$
$q$
$t, \tau$
$t r$
$\omega$
$B . \quad$ Sets
A
$B$
CH

\section{NOMENCLATURE}

Index for assets

Index for day/night time block

Index for EVCS

Indexes for nodes

Index for alternatives

Index for feeder types

Index for distributed generation (DG) types

Index for quarters

Index for time stages

Index for transformers

Index for scenarios

\section{Set of assets, $A=\{L, T R, S S, P, C H\}$}

Set of blocks (day and night)

Set of EVCS types. $C H=\{N C H, A C H\}$ where $N C H$ and $A C H$ denote new and additional capacity for EVCS, respectively

$K^{l}, K^{t r}, K^{p}$ Set of alternatives of feeders, transformers, $K^{S T}, K^{c h}$

\section{DG, ESS and EVCS}

This work was supported in part by the Ministry of Economy and Competitiveness of Spain, under Project ENE2015-63879-R (MINECO/FEDER, UE), and the Junta de Comunidades de Castilla - La Mancha, under Project POII-2014-012-P and Grant PRE2014/8064.

P. Meneses de Quevedo, G. Muñoz-Delgado and J. Contreras are with E.T.S. de Ingenieros Industriales, University of Castilla - La Mancha, 13071 Ciudad Real, Spain (e-mail: pilar.meneses@uclm.es; gregorio.munoz.delgado@gmail.com; Javier.Contreras@uclm.es).
$L$

Set of feeder types.

$L=\{E F F, E R F, N R F, N A F\}$ where $E F F$,

$E R F, N R F$, and $N A F$ denote existing fixed feeders, existing replaceable feeders, new replacement feeders, and newly added feeders

$P \quad$ Set of DG types. $P=\{W, \Theta\}$ where $W$ and $\Theta$ denote wind power and photovoltaic generation (PV), respectively

Set of quarters (4 seasonal quarters)

Set of time stages

$\underset{T}{Q}$

$T R$

Set of transformer types. $T R=\{E T, N T\}$ where $E T$ and $N T$ denote existing transformer and new transformer, respectively

$\Omega^{N}, \Omega_{i}^{l}, \Omega_{t}^{L N}$

$\Omega^{S S}, \Omega^{p}$,

$\Omega^{S T}, \Omega^{\text {ch }}$

Set of system nodes, nodes connected to node $i$ by a feeder of type $l$, load nodes, substation nodes, candidate nodes for DG, candidate nodes for ESS and candidate nodes for EVCS

Set of scenarios

$\Upsilon^{l}$

Set of branches with feeders of type $l$

\section{Parameters}

$C_{k}^{I, l}, C_{i}^{I, S S}, C_{k}^{I, N T}$, Investment cost of feeders, substations, new $C_{k}^{I, p}, C_{k}^{I, S T}, C_{k}^{I, c h}$ transformers, DG, ESS and EVCS

$C_{k}^{M, l}, C_{k}^{M, t r}, C_{k}^{M, p}$ Maintenance cost of feeders, transformers, $C_{k}^{M, S T}, C_{k}^{M, c h} \quad$ DG, ESS and EVCS

$C_{t q b \omega}^{S S}$

$C_{k}^{E, p}$

$C_{k}^{S T, p r o d}$

$C_{k}^{S T, \text { store }}$

$C^{U}$

$D_{i t q b \omega}$

$\operatorname{dem}_{t q b \omega}^{E V}$

$\bar{F}_{i j k}^{l}$

$\bar{G}_{k}^{t r}$

$\bar{G}_{k}^{p}$
$\widehat{G}_{i k t q b \omega}^{p}$

$\underline{G}_{k}^{S T}$

$\overline{\bar{G}}_{k}^{S T}$

$\bar{G}_{k}^{c h}$

I

$I B_{t}$

$l_{i j}$
Cost of the energy purchased at the substation Production cost of DG

Production cost of ESS

Storage cost of ESS

Cost of the unserved energy

Nodal demand

Total EV charging demand needed in the whole system

Upper limit for actual current flows through feeders transformer of type $t r$

Maximum capacity of a generator of type $p$

Maximum power availability of a generator of

type $p$

Minimum capacity of ESS

Maximum capacity of ESS

Maximum capacity of an EVCS

Annual investment rate

Investment limit at stage $t$

Feeder length
Upper limit for energy supplied by a 


\begin{tabular}{|c|c|}
\hline$n_{D G}, n_{T}$ & $\begin{array}{l}\text { Number of candidate nodes for DG and } \\
\text { number of time stages }\end{array}$ \\
\hline$p f$ & System power factor \\
\hline$R R^{a}$ & Capital recovery rates for each asset \\
\hline$Z_{i j k}^{l}$ & $\begin{array}{l}\text { Unitary impedance magnitude of feeder of } \\
\text { type } l\end{array}$ \\
\hline$\pi_{q b \omega}$ & $\begin{array}{l}\text { Weight of scenario } \omega \text { in day/night block } b \text { for } \\
\text { quarter } q\end{array}$ \\
\hline$\Delta_{q b}$ & $\begin{array}{l}\text { Duration in hours of day/night block } b \text { for } \\
\text { quarter } q\end{array}$ \\
\hline$\eta_{i k}^{S T, p r o d}$ & Production efficiency rate for ESS \\
\hline$\eta_{i k}^{S T, \text { store }}$ & Storage efficiency rate for ESS \\
\hline$\varepsilon$ & Penetration limit for generation \\
\hline$v^{a}$ & Lifetime of asset $a$ [number of stages] \\
\hline
\end{tabular}

D. Variables

$c_{t}^{I} \quad$ Investment cost at stage $t$

$c_{t}^{E} \quad$ Energy production cost at stage $t$

$c_{t}^{M} \quad$ Maintenance cost at stage $t$

$c_{t}^{R} \quad$ Energy losses cost at stage $t$

$c_{t}^{U} \quad$ Unserved energy cost at stage $t$

$d_{i t q b \omega}^{c h} \quad$ Charging demand in EVCS at node $i$

$d_{i t q b \omega}^{U} \quad$ Nodal unserved energy

$f_{i j k t q b \omega}^{l} \quad$ Current flow through alternative $k$ of feeder type $l$ installed in branch $i j$ at stage $t$, day/night block $b$ for quarter $q$ and scenario $\omega$, measured at node $i$. It is greater than 0 if node $i$ is the supplier and 0 otherwise $g_{i k t q b \omega}^{p} \quad$ Energy supplied by a generator of type $p$ $g_{i k t q b \omega}^{t r} \quad$ Energy supplied by a transformer of type $t r$

$g_{i k t q b \omega}^{\text {ST,prod }} \quad$ Power production of a ESS

$g_{\text {ST, store }}$

$g_{\text {iktqb } \omega}^{\text {sT }}$

$x_{i t}^{S S}, x_{i k t}^{N T}, x_{i j k t}^{l}$,

$x_{i k t}^{p}, x_{i k t}^{S T}, x_{i k t}^{c h}$

ST,prod

$u_{i k t q b \omega}^{S T, p r o d}$

$u_{\text {ST, store }}$

iktqb $\omega$

$y_{i t}^{S S} y_{i k t}^{N T}, y_{i j k t}^{l}$

$y_{i k t}^{p}, y_{i k t}^{S T}, y_{i k t}^{c h}$

$v_{i t q b \omega}$

\section{Power stored of a ESS}

Binary investment variable for substations, new transformers, feeders, DG, ESS and EVCS

Binary variables related to production and storage of ESS

Binary utilization variable for substations, new transformers, feeders, DG, ESS and EVCS

Nodal voltage

\section{INTRODUCTION}

\section{A. Motivation}

$\mathrm{F}$ UTURE decisions in DSEP models should consider the optimal location and sizing of EVCS, ESS and renewable DG. Their inappropriate location could have negative effects on the distribution system increasing power losses and degrading voltage profiles at some nodes [1]. Recently, EVs have aroused extensive attention due to the growing concerns in greenhouse gas emissions. More and more, EVs will be integrated in electrical power systems through charging facilities. This would generate a significant charging demand with uncertainties that along with the uncertainties arising from renewable energy may create an important challenge to new distribution systems. In order to deal with this problem, the estimation of the additional EV demand has to be done by using a vehicle decision algorithm considering EV travel data based on some assumptions on when EVs need to be charged. Moreover, the value of ESS relies on the existence of additional capacity to the grid, which represents an important issue to the increasing penetration of renewable energy and EV charging demand, while reducing generation costs.

Traditionally, multistage distribution planning models have been used to optimize generation investment meeting the growing demand in a centralized framework. In a more recent context, where renewable energy is increasing, uncertainty has to be considered in the model. Hence, one of the key aspects related to investment decisions is to represent the uncertainty and the correlation of the stochastic information (load, wind speed, solar irradiation, cost of the energy at the substation and EV charging demand). A clustering technique, the $\mathrm{k}$ means ++ algorithm, is used to arrange data in groups according to their similarities, reducing the historical data into a large enough set of clusters. A detailed representation of the whole model is presented in the following sections.

This work stresses the importance of integrating ESS in distribution and generation system expansion planning given that medium-voltage EVCS should be appropriately located and sized in order to minimize the present value of the overall generation and distribution costs.

\section{B. Literature Review}

In the last few years, some models have been developed in distribution system planning within a centralized framework. Conventional planning models have solved the optimal expansion of distribution assets with the replacement and addition of feeders, the reinforcement of existing substations and the construction of new substations [2]. In [3], a review of the state-of-art of DSEP is presented. The increasing penetration of DG, run on renewable energy, requires its incorporation in distribution planning models to decide its location and timing [4]-[7]. Additionally, the uncertainty related to the unpredictability of the RES is commonly considered. In [4] the uncertainties in demand and DG are represented based on the theory of multiple scenarios running a genetic algorithm. In [5], the uncertainty related to load and electricity price is modeled through Monte Carlo simulation. In [6], a scenario-based stochastic programming framework is used to minimize the present value of the expected investment and operational cost in DSEP under correlated uncertainty based on duration curves. Similarly, in [7], demand response and ESS are also included in the distribution and generation expansion planning.

Other works have studied the operational planning of ESS. In [8], the concept of system states as opposed to load level duration curves (as in [6], [7]) is introduced, allowing for a better incorporation of chronological information. In [9], the optimal operation planning of batteries in distribution networks is performed by metaheuristic methods using probabilistic variation of the inputs with the point estimation method for optimal planning of batteries. In [10], a multistage 
expansion planning model for replacing and adding circuits is performed, in which typical daily scenarios are assessed for the hourly economic dispatch of the ESS. Some works include the existence of EV loads in the joint distribution, generation and ESS expansion planning without including the installation cost in EVCS. In [11], a non-parametric chance-constrained optimization to invest in ESS units is proposed, in which the uncertainties of DGs and EVs, were considered using the probability density function. In [12], a multi-objective optimal planning of battery energy storage and DG units in an active distribution network is presented, in which the power profile of the EVs was modeled by fuzzy values.

Recently, more attention has been paid to the optimal planning of EVCS. In [13], the optimal planning of EVCS in distribution systems is developed with the minimization of total costs. In [14], a multi-objective mixed integer non-linear programming planning model is proposed for the new and replaced distribution network assets, EVCS and losses. In [15] a multi-objective planning model of a distribution network containing DG and EVCS is implemented. A scenario expansion planning for distribution systems is proposed in [16] considering the integration of EV, with dumb charging and coordinated charging modes.

In contrast to [10]-[12], our paper considers the investment decisions in EVCS. Additionally, different from [13]-[15], our paper jointly incorporates the expansion planning decision model of the network, DG (both wind and PV) and ESS investments. In [7], the goal is to maximize the net social benefit of the system being represented by the present value of the total payment of the consumers minus the present value of the total costs. In our paper, the objective is also different, as we are minimizing the total costs of expansion planning with the interaction of ESS and EV. Furthermore, in [7] the approach is different due to introduction of demand response related with the management of the active demand. Unlike [6] and [7], the scenarios are obtained using a clustering technique while also including the uncertainty associated with the cost of the energy purchased at the substation. This technique is better suited than the duration curves applied in [7]. Likewise, as said in [19], the k-means++ technique allows representing the operating conditions comparatively with greater accuracy.

\section{Paper Contributions and Organization}

TABLE I

COMPARISON OF THE INVESTMENT DECISIONS PLANNING MODELS

\begin{tabular}{ccccc}
\hline Approach & $\begin{array}{c}\text { Network } \\
\text { Investment }\end{array}$ & $\begin{array}{c}\text { RES } \\
\text { Investment }\end{array}$ & $\begin{array}{c}\text { ESS } \\
\text { Investment }\end{array}$ & $\begin{array}{c}\text { EVCH } \\
\text { Investment }\end{array}$ \\
\hline$[2]$ & $\checkmark$ & $\times$ & $\times$ & $\times$ \\
{$[4-6]$} & $\checkmark$ & $\checkmark$ & $\times$ & $\times$ \\
{$[7]$} & $\checkmark$ & $\checkmark$ & $\checkmark$ & $\times$ \\
{$[9]$} & $\times$ & $\times$ & $\checkmark$ & $\times$ \\
{$[10]$} & $\checkmark$ & $\times$ & $\times$ & $\times$ \\
{$[11]$} & $\times$ & $\times$ & $\checkmark$ & $\times$ \\
{$[12]$} & $\times$ & $\checkmark$ & $\checkmark$ & $\times$ \\
{$[13]$} & $\times$ & $\times$ & $\times$ & $\checkmark$ \\
{$[14]$} & $\checkmark$ & $\times$ & $\times$ & $\checkmark$ \\
{$[15]$} & $\checkmark$ & $\checkmark$ & $\times$ & $\checkmark$ \\
{$[16]$} & $\checkmark$ & $\times$ & $\times$ & $\times$ \\
Proposed approach & $\checkmark$ & $\checkmark$ & $\checkmark$ & $\checkmark$ \\
\hline
\end{tabular}

Table I summarizes the contents of the state-of-art in comparison with our work. Note that, as described in the literature review, some works already consider the existence of ESS, EV and RES without focusing on the corresponding investment decisions.

The main contributions of this paper are:

- The model includes the expansion planning of the distribution system including new and replacement feeders, new substations, additional transformers, RES, ESS, EVCS and additional capacity in the stations, simultaneously. The combination of the investment decisions of all these assets has not been presented yet.

- The prospective model stresses the relevance of integrating ESS in investment models under the increasing penetration of RES and the upcoming integration of EV under uncertainty. The main goal of this paper it is to show and analyze the interaction between ESS and EV.

- A scenario-based stochastic programming model is used to take into account the uncertainty of demand, the cost of the energy purchased at the substation, wind power, PV and EV charging demand.

- The associated deterministic equivalent is formulated as a mixed-integer linear programming model suitable for commercially available software.

The rest of the paper is organized as follows. The methodology to model EV charging demand and scenarios are presented in Section II. In Section III, the stochastic programming model for cost minimization is formulated. In Section IV, the case study and the results are reported. Finally, conclusions are drawn in Section V.

\section{Methodology}

In this section, we describe how to estimate the additional EV demand that needs to be considered in the optimal expansion planning of the whole system and the k-means++ clustering technique used to characterize the uncertain nature of the inputs and their correlation.

\section{A. Modeling for EV Charging Demand}

The increasing number of EVs connected for charging has a significant impact on several power system parameters, such as generation capacity, transformer loading level, line congestion level and load profile. Therefore, the expected increasing penetration of EV calls for the estimation of the additional demand.

We propose a method based on realistic vehicle statistics extracted from the 2009 (US) National Highway Travel Survey (NHTS) data [17] to calculate the total charging demand for all EVs. This survey includes data of each household, type of vehicle, trip distance, start and end time, month, day of the week, trip purpose and the location (city in the US) where these trips take place. A trip is the journey done by a vehicle when it goes from the driver's home to his/her workplace, or a commercial area, and vice versa. Due to the lack of data of EV travel patterns, we assume them to be the same as the traditional fossil-fueled ones.

To estimate the charging demand for EVs, a vehicle decision model has been implemented in MATLAB [18] as presented in Fig. 1. First, we exclude the trips with mileage greater than the maximum mileage driven using electricity. 
The proposed methodology comprises six steps that are described as follows:

1) For each vehicle $v$, a battery capacity is assigned randomly (24 kWh, $30 \mathrm{kWh}, 36 \mathrm{kWh})$. We assume that all EVs are fully charged at the beginning of the first trip.

2) For each vehicle $v$, month $m$ and day $d$, a trip $T$ is assigned.

3) The model checks the state of charge (SOC) of the battery when the vehicle finishes trip $T$.

4) If the SOC does not reach the minimum $(20 \%$ of the battery capacity), the algorithm looks for the next trip computing and updating the charging demand.

5) If the SOC reaches the minimum it is assumed that the vehicle charges its battery prior to that trip $T$ in the interval of time (between trip $T-1$ and $T$ ) that vehicle $v$ is stopped. Then, the SOC of the battery is checked again. In case the minimum is still reached the trip is excluded and the algorithm looks for the next trip.

6) After the last trip the algorithm iteratively runs along days, months and vehicles.

The charging time depends on three factors: battery capacity, SOC and power level of the charger. We consider a normal power supply of $3.3 \mathrm{~kW}$ at the EVCS. Hence, the total EV charging demand for all the vehicles is computed per hour, day, month and time stage (year) for the entire system.

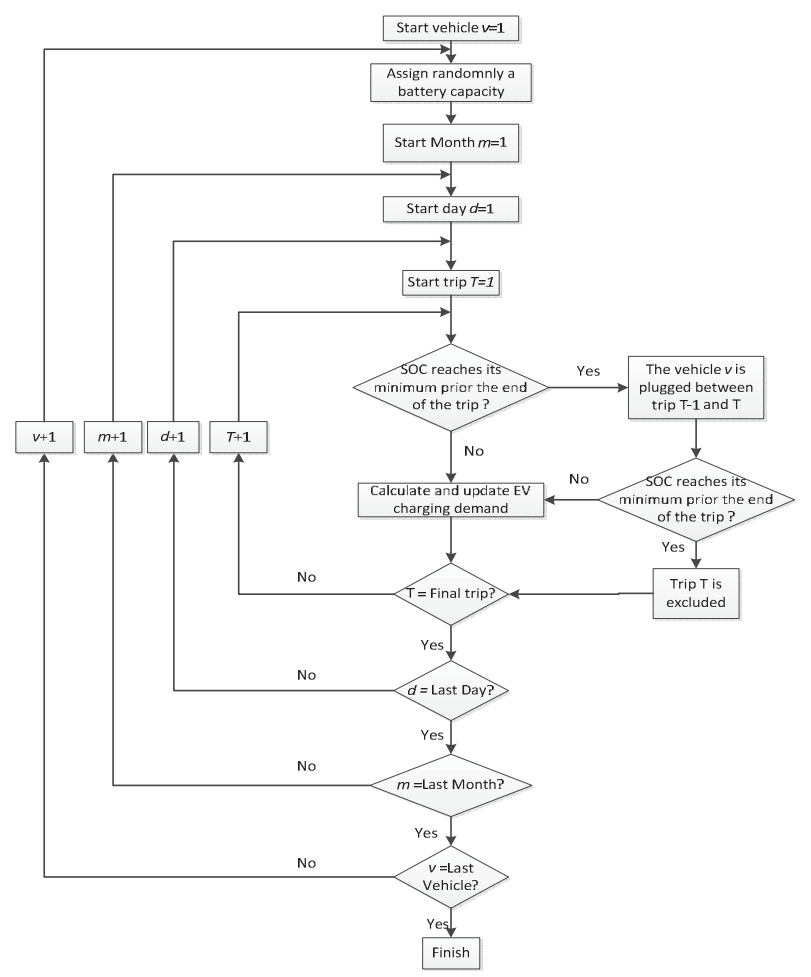

Fig 1. Flowchart of the vehicle decision model.

\section{B. Uncertainty: K-means ++ Method}

The incorporation of renewable DG and EVCS in the multistage expansion planning of distribution systems yields new sources of uncertainty associated with the variability of RES and EV charging demand. Such uncertainty sources along with the conventional uncertainty associated with demand and the cost of energy purchased at the substation are considered. To that end, the variability of load demand, EV charging demand, wind speed, solar irradiation and cost of energy at the substation is characterized through a scenariobased stochastic programming framework. The set of scenarios is built on historical data through the clustering technique shown in [19], allowing to keep the correlation among the initial uncertain data as in [20]

In this paper we use the k-means ++ algorithm from Matlab [18] due to its simplicity and good performance. K-means clustering is based on computational geometry. The goal is to arrange the data into groups according to their similarities. A certain number of centers (centroids) are chosen in order to reduce the initial data based on the mathematics: minimizing the total squared distance between each point and its closest center.

With the purpose of minimizing the loss of information of the sequence between hours, the historical data is split into quarters (winter, spring, summer and autumn) as it is needed for representing the transition function of the ESS. This means it is applied 8 times, for each quarter (winter, spring, summer and autumn) and day/night block, so there are 4 quarters and 2 day/night blocks. Finally, there are 96 (12 clusters per quarter and block $\mathrm{x} 4$ quarters $\mathrm{x} 2$ blocks) operating conditions at each stage representing the different scenarios. In this matrix, each row has 5 columns representing the uncertain parameters: load, EV demand, wind speed, solar irradiation and cost of the energy purchased at the substation.

The k-means methodology is an iterative algorithm. In this paper, specifically, we have used k-means ++ implemented in Matlab R2016b. In data mining, k-means++ is an algorithm to choose the initial values (or "seeds") of the $k$-means clustering algorithm. This algorithm improves the initialization of the centroids before starting the standard kmeans, guaranteeing a faster and better solution [21].

The algorithm works as follows:

Step 1) Select the number of required clusters $K$, according to the needs of the problem. This number should encompass a good representation of the operating conditions of the system.

Step 2) Take one centroid, chosen uniformly at random from initial data set $(x) . D(x)$ denotes the shortest distance from a data point to the closest center, which has been already chosen.

Step 3) Take a new centroid, $c_{i}$, choosing $x$ with probability $\frac{D(x)^{2}}{\sum_{x} D(x)^{2}}$.

Step 4) Repeat step 3 until there are $K$ centers altogether.

Step 5) Compute the distances between each data point and each cluster centroid based on the use of Euclidean distances.

Step 6) Assign each point of the data to the closest centroid based on the use of Euclidean distances.

Step 7) Recalculate the cluster centroids, $c_{i}$, to be the center of mass of all points in $C_{i}$ :

$$
c_{i}=\frac{1}{\left|C_{i}\right|} \sum_{x \in C i} x
$$

Steps 5-7 are performed iteratively until the cluster compositions do not vary between two consecutive iterations. The cluster centroids (matrix of 96 values x 5 parameters) are the output of this algorithm as well as the number of 
observations assigned to each cluster. Each cluster centroid is represented by the values of the cost of the energy purchased at the substation, wind speed, solar irradiation, load and EV charging demand, which show a system operating condition. Nevertheless, the duration of each scenario is determined by the number of observations (each observation lasts one hour) in each cluster.

\section{PROBLEM FORMULATION}

In the multistage expansion planning model, a scenariobased stochastic programming is used to minimize the present value of the expected total cost of the distribution network from a centralized framework viewpoint. The proposed model is built on an associated scenario-based deterministic equivalent formulated as a MILP in which i) the planning horizon is divided into $T$ stages of known duration, ii) radial operation is explicitly imposed, iii) an approximate network model is used, iv) several investment alternatives exist for each asset, and v) a perpetual planning horizon is considered for the operating costs.

\section{A. Objective Function}

The model minimizes the present value of the total expected cost:

$$
\begin{gathered}
\min =\left\{\sum_{t} \frac{(1+I)^{-t}}{I} c_{t}^{I}+\right. \\
+\left[\sum_{t}(1+I)^{-t}\left(c_{t}^{M}+c_{t}^{E}+c_{t}^{R}+c_{t}^{U}\right)+\right. \\
\left.\left.\frac{(1+I)^{-n_{T}}}{I}\left(c_{n_{T}}^{M}+c_{n_{T}}^{E}+c_{n_{T}}^{R}+c_{n_{T}}^{U}\right)\right]\right\}
\end{gathered}
$$

where:

$$
\begin{aligned}
& c_{t}^{I}=\sum_{l \in\{N R F, N A F\}} R R^{l} \sum_{k \in K^{l}} \sum_{(i, j) \in Y^{l}} C_{k}^{I, l} e_{i j} x_{i j k t}^{l}+ \\
& R R^{S S} \sum_{i \in \Omega^{S S}} C_{i}^{I, S S} x_{i t}^{S S}+ \\
& R R^{N T} \sum_{k \in K^{N T}} \sum_{i \in \Omega^{S S}} C_{k}^{I, N T} x_{i k t}^{N T}+ \\
& \sum_{p \in P} R R^{p} \sum_{k \in \mathrm{K}^{p}}^{k \in K_{i \in \Omega^{p}}} \sum_{i \in \Omega^{S S}}^{I, p} p f \bar{G}_{k}^{p} x_{i k t}^{p}+ \\
& R R^{S T} \sum_{k \in \mathrm{K}^{S T}} \sum_{i \in \Omega^{S T}} C_{k}^{I, S T} p f \bar{G}_{k}^{S T} x_{i k t}^{S T}+ \\
& \sum_{c h \in C H} R R^{c h} \sum_{k \in \mathrm{K}^{c h}} \sum_{i \in \Omega^{c h}}^{k \in \mathrm{K}^{s}} C_{k}^{I, c h} p f \bar{G}_{k}^{c h} x_{i k t}^{c h} ; \quad \forall t \in T \\
& c_{t}^{M}=\sum_{l \in L} \sum_{k \in K^{l}}^{k \in K^{c h}} \sum_{i, j) \in \Upsilon^{l}} C_{k}^{M, l}\left(y_{i j k t}^{l}+y_{j i k t}^{l}\right)+ \\
& \sum_{t r \in T R} \sum_{k \in K^{t r}} \sum_{i \in \Omega^{S S}} C_{k}^{M, t r} y_{i k t}^{t r}+\sum_{p \in P} \sum_{k \in K^{p}} \sum_{i \in \Omega^{p}} C_{k}^{M, p} y_{i k t}^{p}+ \\
& \sum_{k \in \mathrm{K}^{S T}} \sum_{i \in \Omega^{S T}} C_{k}^{M, S T} y_{i k t}^{S T}+\sum_{\substack{c h \in C H \\
\forall t \in T}} \sum_{k \in \mathrm{K}^{c h}} \sum_{i \in \Omega^{c h}} C_{k}^{M, c h} y_{i k t}^{c h} ;
\end{aligned}
$$

$$
\begin{aligned}
& \left.\sum_{l \in L} \sum_{k \in K^{l}} \sum_{(i, j) \in Y^{l}} Z_{k}^{l} \ell_{i j}\left(f_{i j k t q b \omega}^{l}+f_{j i k t q b \omega}^{l}\right)^{2}\right) ; \forall t \in T \\
& c_{t}^{U}=\sum_{q \in Q} \sum_{b \in B} \sum_{\omega \in \Pi} \pi_{q b \omega} \Delta_{q b} C^{U} p f \sum_{i \in \Omega_{t}^{L N}} d_{i t q b \omega}^{U} ; \forall t \in T
\end{aligned}
$$

The objective function (1) contains three terms. The first one corresponds to the present worth value of the investment cost under the assumption of a perpetual or infinite planning horizon [22]. That is, the investment cost is amortized annually throughout the lifetime of the assets, considering that after their lifetime has expired there is a reinvestment in an identical equipment. The second term is the present value of the operating costs throughout the time stages. Lastly, the third term shows the present value of the operating costs incurred after the last time stage assuming a perpetual planning.

The total cost (1) comprises five different costs, namely investment cost (2), maintenance cost (3), production cost (4), cost of energy losses (5), and unserved energy costs (6). The capital recovery rates for investing in feeders, new transformers, substations, generators, ESS and EVCS are calculated in a generic form as $R R^{a}=I(1+I)^{v^{a}} /$ $\left((1+I)^{v^{a}}-1\right)$, which are dependent on the lifetime of each asset. It is noticeable that two binary variables are associated with each feeder in order to model its utilization in the directions, $y_{i j k t}^{l}$ and $y_{j i k t}^{l}$. The same occurs with the current flow through the feeder. The energy losses are formulated as quadratic terms and are linearized approximating them by a set of tangent lines. This can be seen in section III.E in [6].

\section{B. Constraints}

Firstly, the constraints related with the technical operation of the system such as Kirchhoff Laws and operational limits are formulated. In (7) the nodal current flow balance equation is stated. In (8) the Kirchhoff Voltage Law for all feeders is represented. Note this constraint includes nonlinearities which are recast as linear expressions as provided in section III.E in [6]. Constraint (9) sets the bounds on the magnitudes of nodal voltages. In (10)-(13), the upper and lower limits on the current flow and generation power are represented. In (14), the 
level of penetration of DG is limited as a fraction $\varepsilon$ of the load and charging demand.

$$
\begin{aligned}
& \sum_{l \in L} \sum_{k \in K^{l}} \sum_{j \in \Omega_{i}^{l}}\left(f_{i j k t q b \omega}^{l}-f_{j i k t q b \omega}^{l}\right) \\
& =\sum_{t r \in T R} \sum_{k \in K^{t r}} g_{i k t q b \omega}^{t r}+\sum_{p \in P} \sum_{k \in K^{p}} g_{i k t q b \omega}^{p}+ \\
& \sum_{k \in K^{s t}} g_{i k t q b \omega}^{S T, p r o d}-\sum_{k \in K^{S t}} g_{i k t q b \omega}^{\text {ST,store }}-D_{i t q b \omega}-d_{i t q b \omega}^{U} \\
& -d_{i t q b \omega}^{C H} ; \forall i \in \Omega^{N}, \forall t \in T, \forall q \in Q, \forall b \in B, \forall \omega \in \Pi \\
& y_{i j k t}^{l}\left[Z_{i j k}^{l} \ell_{i j} f_{i j k t q b \omega}^{l}-\left(v_{i t q b \omega}-v_{j t q b \omega}\right)\right]=0 \text {; } \\
& \forall l \in L, \forall i \in \Omega_{j}^{l}, \forall j \in \Omega^{N}, \forall k \in K^{l}, \forall t \in T, \forall q \in Q, \\
& \forall b \in B, \forall \omega \in \Pi \\
& \underline{V} \leq v_{i t q b \omega} \leq \bar{V} \\
& \forall i \in \Omega^{N}, \forall t \in T, \forall q \in Q, \forall b \in B, \forall \omega \in \Pi \\
& 0 \leq f_{i j k t q b \omega}^{l} \leq y_{i j k t}^{l} \bar{F}_{i j k}^{l} ; \\
& \forall l \in L, \forall i \in \Omega_{j}^{l}, \forall j \in \Omega^{N}, \forall k \in K^{l}, \forall t \in T, \forall q \in Q \\
& , \forall b \in B, \forall \omega \in \Pi \\
& 0 \leq g_{i k t q b \omega}^{t r} \leq y_{i k t}^{t r} \bar{G}_{k}^{t r} \\
& 0 \leq g_{i k t q b \omega}^{p} \leq y_{i k t}^{p} \widehat{G}_{i k t q b \omega}^{p} ; \\
& 0 \leq d_{i t q b \omega}^{U} \leq d_{i t q b \omega}+d_{i t q b \omega}^{C H} ; \\
& i \in \Omega^{N}, \forall t \in T, \forall q \in Q, \forall b \in B, \forall \omega \in \Pi \\
& \sum_{p \in P} \sum_{k \in K^{p}} \sum_{i \in \Omega^{p}} g_{i k t q b \omega}^{p}+\sum_{k \in K^{S T}} \sum_{i \in \Omega^{S T}}\left(g_{i k t q b \omega}^{S T, p r o d}-g_{i k t q b \omega}^{S T, \text { store }}\right) \\
& \leq \varepsilon\left(\sum_{i \in \Omega_{t}^{L N}} D_{i t q b \omega}+d_{i t q b \omega}^{C H}\right) \\
& \forall t \in T, \forall b \in B, \forall q \in Q, \forall \omega \in \Pi
\end{aligned}
$$

The power supplied or stored by an ESS unit is bounded between the lower and upper capacity values, (15)-(16). When the ESS unit is used, to avoid simultaneous production and storage, two binary variables are defined in (17).

$$
\begin{gathered}
G_{k}^{S T} u_{i k t q b \omega}^{S T, p r o d} \leq g_{i k t q b \omega}^{S T, p r o d} \leq \bar{G}_{k}^{S T} u_{i k t q b \omega}^{S T, p r o d} ; \\
\forall i \in \Omega^{S T}, \forall k \in K^{S T}, \forall t \in T, \forall q \in Q, \forall b \in B \forall \omega \in \Pi \\
G_{k}^{S T} u_{i k t q b \omega}^{S T, \text { store }} \leq g_{i k t q b \omega}^{S T, \text { store }} \leq \bar{G}_{k}^{S T} u_{i k t q b \omega}^{S T, \text { store }} ; \\
\forall i \in \Omega^{S T}, \forall k \in K^{S T}, \forall t \in T, \forall q \in Q, \forall b \in B, \\
, \forall q \in Q, \forall \omega \in \Pi \\
u_{i k t, p r o d}^{S T}+u_{i k t q b \omega}^{S T, \text { store }} \leq y_{i k t}^{S T} ; \\
\forall i \in \Omega^{S T}, \forall k \in K^{S T}, \forall t \in T, \forall q \in Q, \forall b \in B, \\
\forall q \in Q, \forall \omega \in \Pi
\end{gathered}
$$

The utilization and investment constraints follow, in which a maximum of one reinforcement, replacement or addition is allowed for each system asset and location along the planning horizon, (18)-(23). In (24)-(25), new transformers can be added at substations as well as additional capacity in the EVCS, only if they have been built in any previous stage.

$$
\begin{gathered}
\sum_{t \in T} \sum_{k \in K^{l}} x_{i j k t}^{l} \leq 1 ; \forall l \in\{N R F, N A F\}, \forall(i, j) \in \Upsilon^{l} \\
\sum_{t \in T} x_{i t}^{S S} \leq 1 ; \forall i \in \Omega^{S S} \\
\sum_{t \in T} \sum_{k \in K^{N T}} x_{i k t}^{N T} \leq 1 ; \forall i \in \Omega^{S S} \\
\sum_{t \in T} \sum_{k \in K^{p}}^{p} x_{i k t}^{p} \leq 1 ; \forall p \in P, \forall i \in \Omega^{p} \\
\sum_{t \in T} \sum_{t \in T} x_{k \in K^{c h}}^{S T} x_{i k t}^{c h} \leq 1 ; \forall i \in \Omega^{S T} \\
x_{i k t}^{N T} \leq \sum_{\tau=1}^{t} x_{i \tau}^{S S} ; \forall i \in \Omega^{S S}, \forall k \in K^{N T} ; \forall t \in T \\
x_{i k t}^{A C H} \leq \sum_{\tau=1}^{t} x_{i k \tau}^{N C H} ; \forall i \in \Omega^{A C H}, \forall k \in K^{A C H} ; \forall t \in T
\end{gathered}
$$

As seen in [6] and [7], we consider radial operation and meshed topologies. Equations (26)-(28) model the utilization of existing and new feeders indicating the direction of the current flows. Equation (28) model the utilization of the existing replaceable feeders while explicitly characterizing the direction of the current flows. These feeders can undergo replacement so that their utilization is subject to the installation of new replacement feeders, $x_{i j k t}^{N R F}$. Therefore, if an existing replaceable feeder is replaced by a new replacement feeder, i.e., $x_{i j k t}^{N R F}=1$; the utilization of such existing replaceable feeder is disable with constraint (28).

The utilization of new transformers, installed generators, ESS, EV and EVCS are defined in (26)-(32).

$$
\begin{gathered}
y_{i j k t}^{E F F}+y_{j i k t}^{E F F} \leq 1 ; \forall(i, j) \in \Upsilon^{E F F}, \forall k \in K^{E F F}, \forall t \in T \\
y_{i j k t}^{l}+y_{j i k t}^{l} \leq \sum_{\tau=1}^{t} x_{i j k \tau}^{l} ; \\
\forall l \in\{N R F, N A F\}, \forall(i, j) \in \Upsilon^{l}, \forall k \in K^{l}, \forall t \in T \\
y_{i j k t}^{E R F}+y_{j i k t}^{E R F} \leq 1-\sum_{\tau=1}^{t} \sum_{\kappa \in K^{N R F}} x_{i j k \tau}^{N R F} ; \\
\forall(i, j) \in \Upsilon^{E R F}, \forall k \in K^{E R F}, \forall t \in T \\
y_{i k t}^{N T} \leq \sum_{\tau=1}^{t} x_{i k \tau}^{N T} ; \forall i \in \Omega^{S S}, \forall k \in K^{N T}, \forall t \in T \\
y_{i k t}^{p} \leq \sum_{\tau=1}^{t} x_{i k \tau}^{p} ; \forall p \in P, \forall i \in \Omega^{p}, \forall k \in K^{p}, \forall t \in T \\
y_{i k t}^{S T} \leq \sum_{\tau=1}^{t} x_{i k \tau}^{S T} ; \forall i \in \Omega^{S T}, \forall k \in K^{S T}, \forall t \in T \\
y_{i k t}^{c h} \leq \sum_{\tau=1}^{t} x_{i k t}^{c h} ; \forall c h \in C H, \forall i \in \Omega^{c h}, \forall k \in K^{c h}, \\
\forall t \in T
\end{gathered}
$$

In (33), the total investment cost at each stage is limited by a maximum budget. 


$$
\begin{gathered}
\sum_{l \in\{N R F, N A F\}} \sum_{k \in K^{l}} \sum_{(i j) \in Y^{l}} C_{k}^{I, l} e_{i j} x_{i j k t}^{l}+\sum_{i \in \Omega^{S S}} C_{i}^{I, S S} x_{i t}^{S S}+ \\
\sum_{k \in K^{N T}} \sum_{i \in \Omega^{S S}} C_{k}^{I, N T} x_{i k t}^{N T}+\sum_{p \in P} \sum_{i \in \Omega^{p}} C_{k}^{I, p} p f \bar{G}_{k}^{p} x_{i k t}^{p}+ \\
\sum_{p \in P} \sum_{i \in \Omega^{S T}} C_{k}^{I, S T} p f \bar{G}_{k}^{S T} x_{i k t}^{S T} \\
+\sum_{c h \in C H} \sum_{i \in \Omega^{C H}} C_{k}^{I, c h} p f \bar{G}_{k}^{c h} x_{i k t}^{c h} \leq I B_{t} ; \forall t \in T
\end{gathered}
$$

In addition, in order to have a radial operation, equation (34) sets load nodes to have one input flow. In (35) the rest of the nodes are limited by a maximum of one input flow. Constraints (36)-(43) avoid isolated generators by modeling a fictitious system with fictitious demands as done in [6].

$$
\begin{aligned}
& \sum_{l \in L} \sum_{i \in \Omega_{j}^{l}} \sum_{k \in K^{l}} y_{i j k t}^{l}=1 ; \forall j \in \Omega_{t}^{L N}, \forall t \in T \\
& \sum_{l \in L} \sum_{i \in \Omega_{j}^{l}} \sum_{k \in K^{l}} y_{i j k t}^{l} \leq 1 ; \forall j \notin \Omega_{t}^{L N}, \forall t \in T \\
& \sum_{l \in L} \sum_{k \in K^{l}} \sum_{j \in \Omega_{i}^{l}}\left(\tilde{f}_{i j k t}^{l}-\tilde{f}_{j i k t}^{l}\right)=\tilde{g}_{i t}^{S S}-\widetilde{D}_{i t} ; \forall i \in \Omega^{N}, \forall t \\
& \in T \\
& 0 \leq \tilde{f}_{i j k t}^{E F F} \leq n_{D G} ; \forall i \in \Omega_{j}^{E F F}, \forall j \in \Omega^{N}, \forall k \in K^{E F F}, \forall t \\
& \in T \\
& 0 \leq \tilde{f}_{i j k t}^{E R F} \leq\left(1-\sum_{\tau=1}^{t} \sum_{\kappa \in K^{N R F}} x_{i j k \tau}^{N R F}\right) n_{D G} ; \\
& \forall(i, j) \in \Upsilon^{E F F}, \forall k \in K^{E R F}, \forall t \in T \\
& 0 \leq \tilde{f}_{j i k t}^{E R F} \leq\left(1-\sum_{\tau=1}^{t} \sum_{\kappa \in K^{N R F}} x_{i j \kappa \tau}^{N R F}\right) n_{D G} ; \\
& \forall(i, j) \in \Upsilon^{E F F}, \forall k \in K^{E R F}, \forall t \in T \\
& 0 \leq \tilde{f}_{i j k t}^{l} \leq\left(\sum_{\tau=1}^{t} x_{i j k \tau}^{l}\right) n_{D G} ; \\
& \forall l \in\{N R F, N A F\}, \forall(i, j) \in \Upsilon^{l}, \forall k \in K^{l}, \forall t \in T \\
& 0 \leq \tilde{f}_{j i k t}^{l} \leq\left(\sum_{\tau=1}^{t} x_{i j k \tau}^{l}\right) n_{D G} ; \\
& \forall l \in\{N R F, N A F\}, \forall(i, j) \in \Upsilon^{l}, \forall k \in K^{l}, \forall t \in T \\
& 0 \leq \tilde{g}_{i t}^{S S} \leq n_{D G} ; \forall i \in \Omega^{S S}, \forall t \in T \\
& \widetilde{D}_{i t}=\left\{\begin{array}{l}
1 ; \quad \forall i \in \Omega_{t}^{L N}, \forall t \in T \\
0 ; \quad \forall i \notin \Omega_{t}^{L N}, \forall t \in T
\end{array}\right.
\end{aligned}
$$

In the case of considering that future networks had bidirectional flows and meshed operation were allowed, equations (34)-(35) should be deleted. However, when considering bidirectional flows and radial operation, equations (34)-(35) of the proposed paper should be replaced with equations (13)-(17) from [23]. These five equations should be adapted to the proposed multistage expansion planning model. Equations (34)-(35) impose the radiality condition per node, while equations (13)-(17) from [23] impose a general radiality condition for the whole network, where upstream flows are allowed.

ESS are good alternatives to integrate large amounts of intermittent renewable energy and improve network reliability because they are more efficient economically and avoid network oversizing. In medium- and long-term planning models, data has to be arranged in scenarios where chronological information is missing, the same as the transition function between hours. Similar to [7], we propose a representation of the transition function (44) to be achieved at the $b$ level (day/night) in each quarter $q$, not being possible to exchange power between quarters.

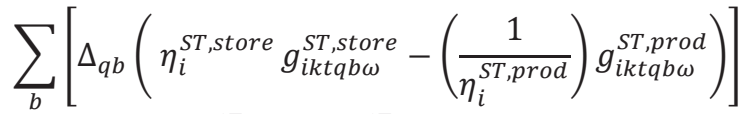

$$
\begin{aligned}
& =0 ; \forall i \in \Omega^{S T}, \forall k \in K^{S T}, \forall t \in T, \forall q \in Q, \forall \omega \in \Pi
\end{aligned}
$$

In this context, for each quarter $q$, the energy can be exchanged (in some scenarios, the ESS produces or stores energy) between operating conditions of the same block $b$ (day or night) and between day and night blocks. This can be seen in Fig. 2, in which there are 12 operating conditions in each block and the sum of the energy produced and stored by the ESS is equal to 0 . Thus, in some operating conditions the demand increases and in other decreases. Note that each operating condition has a different duration with its corresponding scenario probability.

As mentioned in Subsection II.A, the additional demand needed for EVs in the distribution network, $d e m_{t b \omega}^{C H}$, is calculated previously. Equation (45) satisfies the EVCS capacity limits of the charging demand required at the location nodes. Finally, in (46), the sum of the charging demands at all nodes where the EVCS is installed should satisfy the total EV demand needed for the whole system.

$$
0 \leq d_{i t q b \omega}^{c h} \leq \bar{G}_{k}^{c h} y_{i k t}^{c h} ;
$$

$\forall c h \in C H, \forall i \in \Omega^{c h}, \forall k \in K^{c h}, \forall t \in T, \forall b \in B$,

$$
\begin{aligned}
\sum_{c h \in C H} \sum_{i \in \Omega^{C H}} d_{i t q b \omega}^{c h} & =\operatorname{dem}_{t q b \omega}^{E V} ; \forall t \in T, \forall b \in B, \\
\forall q \in Q, \forall \omega \in \Pi & \text { Demand increase }
\end{aligned}
$$

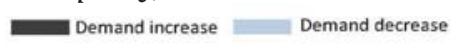

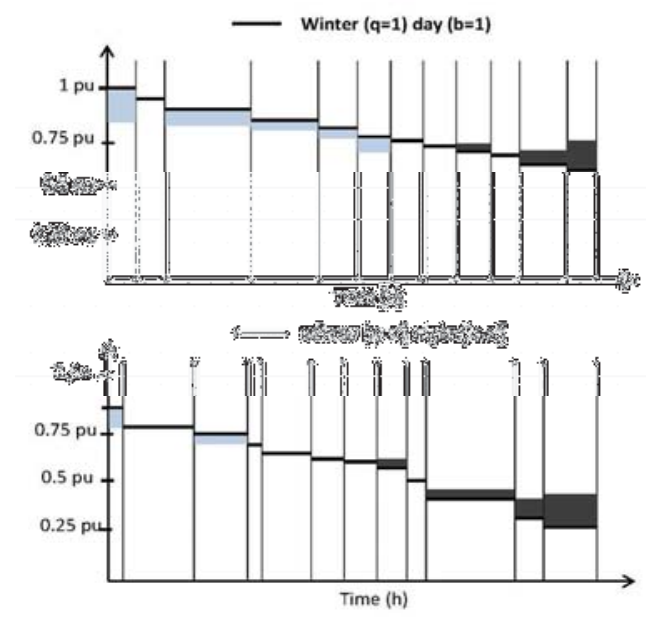

Fig 2. ESS effect on a particular load duration curve. 
All the cases have been solved using MATLAB R2012a [18] and CPLEX 12.6 under GAMS 24.7 [25] on a SIE LADON with two Intel Xeon E5-2698 v3 processors clocking at $2.30 \mathrm{GHz}$ and $256 \mathrm{~GB}$ of RAM. The stopping criterion is based on an optimality gap of $1 \%$. The computation time for each case study is $455 \mathrm{~s}, 968 \mathrm{~s}$ and $12840 \mathrm{~s}$, respectively.

\section{A. Results without ESS and EV Charging Demand}

In Fig. 4 the DSEP is shown with the corresponding replaced and added branches (including the time stage at which this takes place), RES location and new substations and transformers. Alternative 1 is selected by the model for renewable generation and branches. For the sake of simplicity, this has not been illustrated. This is common among all the cases. It is noticeable that all the candidate branches subject to replacement are replaced at stage 1 . In addition, the alternative selected for the new substations is also represented in Fig. 4. We can observe that transformer 1 (10 MVA of capacity) and 2 (15 MVA of capacity) are required for new substations at nodes 53 and 54, respectively.

Table II depicts the stage at which the investment decisions and the node where wind generation, PV generation, new substations are built and the substations are expanded with new transformers. Note that the new substations are required at stages 1 and 2 .

It is noticeable the high investment in renewables, in order to reduce the costs related to production and energy losses, as we allow a $40 \%$ penetration of DG and ESS. In Table III, the numerical disaggregated costs are presented, where the highest investment cost is at stage 4 corresponding to the construction of 3 wind units and $3 \mathrm{PV}$ units as seen in Table II.

TABLE II

INVESTMENT DECISIONS LOCATION IN THE 15-YEAR EXPANSION PLANNING WITHOUT ESS AND EVCS

\begin{tabular}{cccccc}
\hline Investment & Stage 1 & Stage 2 & Stage 3 & Stage 4 & Stage 5 \\
\hline PV & & & $12,30,35$ & $8,20,37$ & 2,22 \\
Wind & $3,10,16$ & 24 & 44 & $38,42,47$ & \\
Transformers & 54 & 53 & & & \\
\hline
\end{tabular}

TABLE III

INVESTMENT AND OPERATIONAL COSTS IN THE 15-YEAR EXPANSION PLANNING WITHOUT ESS AND EVCS $\left(10^{6} \$\right)$

\begin{tabular}{ccccccc}
\hline Costs & Stage 1 & Stage 2 & Stage 3 & Stage 4 & Stage 5 & Total \\
\hline Investment & 17.6 & 6.1 & 12.7 & 18.2 & 4.1 & 58.8 \\
Maintenance & 1.7 & 2.0 & 3.0 & 4.3 & 45.6 & 56.6 \\
Production & 38.1 & 46.4 & 55.5 & 60.4 & 737.1 & 937.4 \\
Energy losses & 1.7 & 2.1 & 2.6 & 3.0 & 40.8 & 50.2 \\
Unserved energy & 0 & 0 & 0 & 0 & 0 & 0 \\
Total & 59.0 & 56.7 & 73.9 & 85.9 & 827.6 & 1103.0 \\
\hline
\end{tabular}

\section{B. Results with EV Charging Demand}

The topology in this situation, including also the EVCS, is shown in Figure 5. We can see that it has also changed slightly. Branch 1-9 (from node 1 to node 9) is replaced at stage 3 . The other replacement candidate branches, as in case A, are replaced at stage 1 .

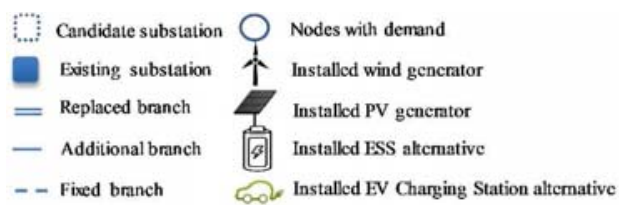

Fig 3. Legend of the on-line diagram of the network.

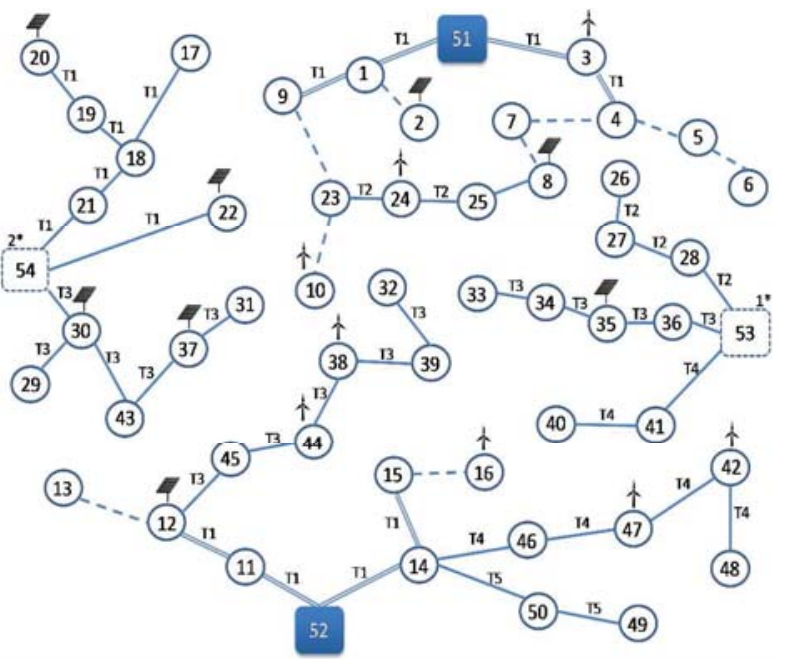

Fig 4. DSEP with network and RES assets.

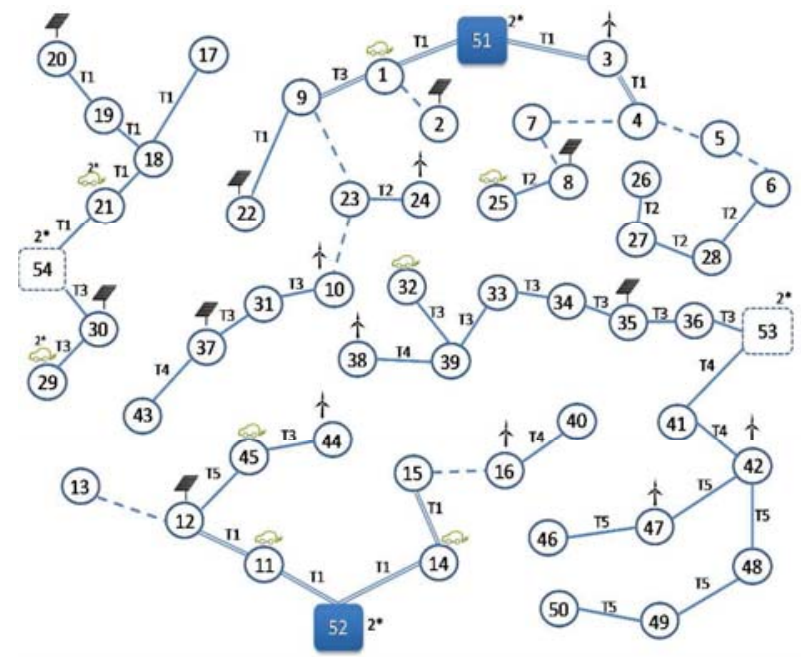

Fig 5. DSEP with network, renewable and EVCS assets.

In Tables IV both the location nodes and the stage of the investment decisions with EVCS are represented. In this case, the substation at node 53 delays its investment to stage 3 . In addition to the increase in the total demand in the system, additional transformers are needed for the existing substations at nodes 51 and 52 (alternative 2 in both cases). Alternative 1 (1 MVA of capacity) is used for all EVCS, except for the one used at node 32. The EVCS located at nodes 21 and 29, constructed at stage 3 , are expanded at stage 4 with alternative 2 (0.75 MVA of capacity).

Including the additional EVs demand in the model, we can see in Table $\mathrm{V}$ that every cost is higher than in case $\mathrm{A}$, as expected. Furthermore, PV investment is slightly accelerated and more transformers are needed in comparison to case A resulting a high investment cost at stage 3 .

$$
\text { TABLE IV }
$$

InVESTMENT DECISIONS LOCATION IN THE 15-YeAR EXPANSION PLANNING WITH EVCS

\begin{tabular}{cccccc}
\hline Investment & Stage 1 & Stage 2 & Stage 3 & Stage 4 & Stage 5 \\
\hline PV & & & $8,12,30,35$ & $20,22,37$ & 2 \\
Wind & $3,10,16$ & 24 & 44 & 38,42 & 47 \\
Transformers & 54 & & 53 & 51 & 52 \\
EVCS & 21 & & $29,32,45$ & $21,25,29$ & $1,11,14$ \\
\hline
\end{tabular}


1

2

3
TABLE V

INVESTMENT AND OPERATIONAL COSTS IN THE 15-YEAR EXPANSION PLANNING WITH EVCS $\left(10^{6} \$\right)$

\begin{tabular}{ccccccc}
\hline Costs & Stage 1 & Stage 2 & Stage 3 & Stage 4 & Stage 5 & Total \\
\hline Investment & 18.2 & 4.7 & 18.1 & 16.9 & 7.9 & 65.8 \\
Maintenance & 1.7 & 1.9 & 3.4 & 4.5 & 50.0 & 61.4 \\
Production & 38.6 & 47.5 & 58.5 & 65.9 & 834.7 & 1045.2 \\
Energy losses & 1.8 & 2.9 & 3.4 & 3.6 & 47.1 & 58.8 \\
Unserved energy & 0 & 0 & 0 & 0 & 0 & 0 \\
Total & 60.2 & 57.1 & 83.4 & 90.9 & 939.6 & 1231.2 \\
\hline
\end{tabular}

\section{Results with ESS and EV Charging Demand}

In this situation we add the possibility to invest in ESS units. The network configuration and new location of the assets is illustrated in Fig 6.

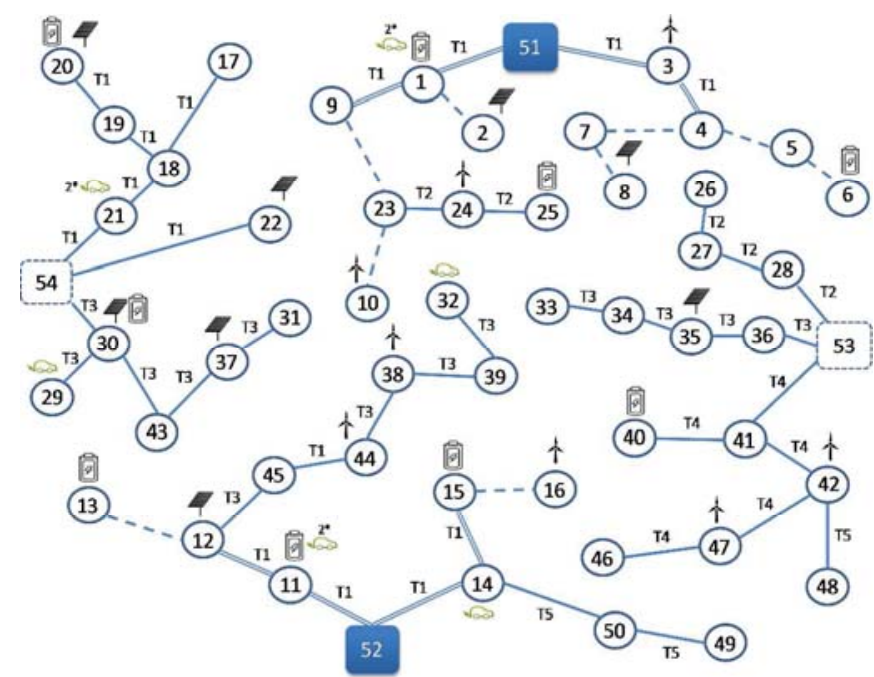

Fig. 6. DSEP with network, renewable, ESS and EVCS assets.

In Table VI, the locations and stages are presented. The renewable investment decision is similar to case B. Note that more ESS units are required at stage 5. The first alternative of the ESS is chosen for all the nodes in which there is a decision to invest in this technology. Moreover, no additional transformer is required in this case. The EVCS investment decision has changed in some stages compared to the previous case. Now, all the EVCS provided by the model are built with alternative 1 ( 3 MVA of capacity). However, at stage 5, the expansion in the EVCS located at nodes 11 and 21 takes place using alternative 2 only ( 0.75 MVA of capacity), being the same alternative provided by the model as in case B.

The costs are presented in Table VII. The energy purchased and energy losses costs are reduced due to existence of ESS. The overall cost is reduced by $3.8 \%$ considering the possibility to invest in storage technologies in comparison to case B. The existence of EV charging demand in the system stresses the importance of investing in this storage units.

The interaction of ESS with EV accelerates the investment in wind and the construction of a new substation. On the other hand, the investment in ESS avoids and defers the investment in new transformers in the existing substations. The effect of the ESS with EV is that the production and energy loss costs decrease, enhancing the total cost for the planner. The main conclusion is that integrating EVs in the distribution system makes the investment in ESS more profitable.
TABLE VI

INVESTMENT DECISIONS LOCATION IN THE 15-YEAR EXPANSION PLANNING

\begin{tabular}{cccccc}
\multicolumn{5}{c}{ WITH ESS AND EVCS } \\
\hline Investment & Stage 1 & Stage 2 & Stage 3 & Stage 4 & Stage 5 \\
\hline PV & & & $12,20,30,35$ & $2,8,37$ & 22 \\
Wind & $3,10,16$ & 24 & 38,44 & 42 & 47 \\
Storage & & 1 & 30 & & $6,11,13,15$ \\
Transformers & 54 & 53 & & & $20,25,40$ \\
EVCS & 11 & & $21,29,32$ & 14 & $1,11,21$ \\
\hline
\end{tabular}

TABLE VII

INVESTMENT AND OPERATIONAL COSTS IN THE 15-YEAR EXPANSION PLANNING WITH ESS AND EVCS $\left(10^{6} \$\right)$

\begin{tabular}{ccccccc}
\hline Costs & Stage 1 & Stage 2 & Stage 3 & Stage 4 & Stage 5 & Total \\
\hline Investment & 18.2 & 11.6 & 22.5 & 11.3 & 16.3 & 79.9 \\
Maintenance & 1.7 & 2.6 & 4.3 & 4.7 & 60.5 & 73.8 \\
Production & 38.6 & 45.9 & 55 & 63.6 & 780.3 & 983.4 \\
Energy losses & 1.7 & 2.2 & 2.6 & 3.0 & 39.7 & 49.2 \\
Unserved energy & 0 & 0 & 0 & 0 & 0 & 0 \\
Total & 60.2 & 62.3 & 84.4 & 82.6 & 896.8 & 1186.3 \\
\hline
\end{tabular}

A sensitivity analysis has been performed with different values of the charging rate of the ESS, as shown in Fig. 7 and different penetration levels of DG with two different situations of the number of candidate buses for the placement of the assets investment, as seen in Fig. 8. Note that a less restrictive charging rate decreases the total investment and operational cost. Besides, the total cost of investment and operational costs decreases as the level of penetration in DG and the number of candidate buses for assets' placement increases.

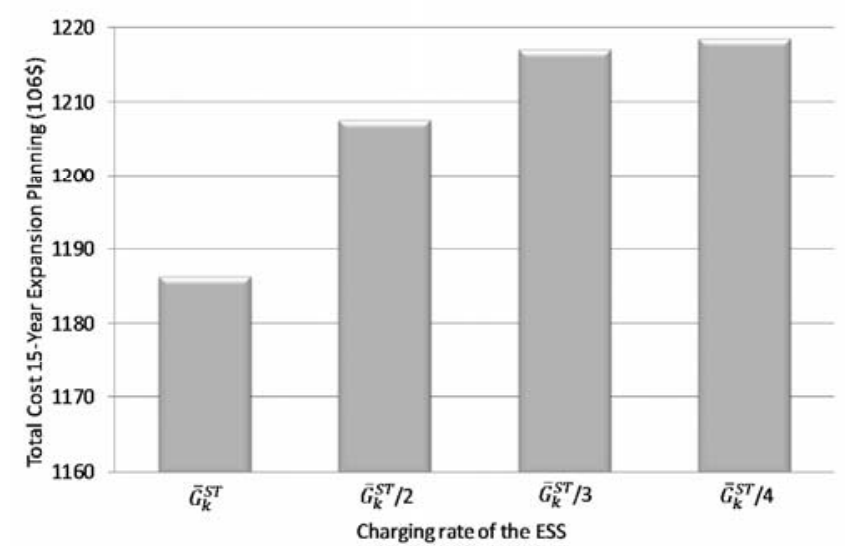

Fig. 7. Total cost of investment and operational costs in the 15-year expansion planning with ESS and EVCS vs. charging rate of the ESS.

Finally, in order to demonstrate and justify the effectiveness of using 96 operating conditions we have simulated other cases with different number of operating conditions for case study $\mathrm{C}$, as seen in Table VIII.

Table VIII shows that the absolute value of the difference of the total cost with $24,48,192,288,384$ and 480 scenarios minus the total cost of with 96 scenarios (base-case) decreases when selecting more scenarios. 


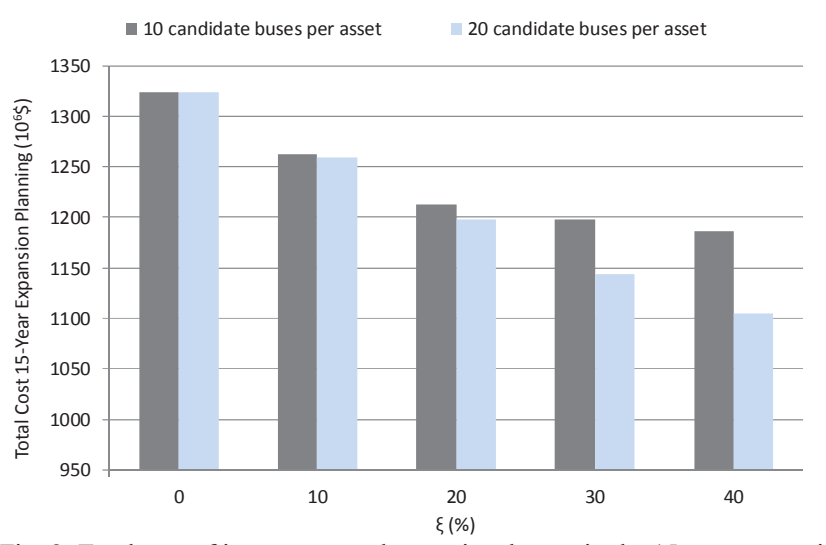

Fig. 8. Total cost of investment and operational costs in the 15-year expansion planning with ESS and EVCS vs. penetration level of DG and ESS.

$$
\text { TABLE VIII }
$$

TOTAL COSTS IN THE 15-YEAR EXPANSION PLANNING WITH ESS AND EV AS A FUNCTION OF THE NUMBER OF OPERATING CONDITIONS

\begin{tabular}{cccc}
\hline Operating conditions & Total cost & $\begin{array}{c}\text { Absolute value of } \\
\text { the difference in (\%) }\end{array}$ & CPU time (s) \\
\hline 24 & 1106.7 & $6.71 \%$ & 1521 \\
48 & 1151.3 & $2.95 \%$ & 4333 \\
96 & 1186.3 & -- & 7908 \\
192 & 1193.4 & $0.60 \%$ & 45070 \\
288 & 1181.9 & $0.37 \%$ & 94719 \\
384 & 1190.7 & $0.37 \%$ & 177422 \\
480 & 1182.3 & $0.33 \%$ & 363339 \\
\hline
\end{tabular}

\section{CONCLUSiOnS}

In this paper, we have presented a MILP model to address the incorporation of investment decisions in recent elements, as EVs and ESS, which will have an important impact in the planning and expansion of future distribution networks.

We have proposed a clustering technique in order to correlate uncertain inputs associated to the stochastic nature of the problem. The scenarios are used in a decision making problem which determines the location of the facilities. Moreover, the EV charging demand has been modeled through a novel EV algorithm. The proposed methodology has been validated with a 54-node network.

We have shown how ESS can contribute to integrate renewable generation and EV charging demand in the coordinated expansion planning of a distribution network minimizing investment and operational costs, avoiding the need to expand the existing substations. Increasing the demand while reducing the energy costs outlines the potential of the combination of EVCS and ESS in distribution networks with a high penetration of renewables.

Future work will consider the possibility that EVs can inject power into the network when they are not in use. In addition, more generation technologies will be added to the model.

\section{REFERENCES}

[1] M. Etezadi-Amoli, K. Choma, and J. Stefani, "Rapid-charge electricvehicle stations," IEEE Trans. Power Del., vol. 25, no. 3, pp. 1883-1887, Jul. 2010.

[2] P.C. Paiva, H.M. Khodr, J.A. Domínguez-Navarro, J.M. Yusta, and A.J. Urdaneta, "Integral planning of primary-secondary distribution systems using mixed integer linear programming," IEEE Trans. Power Syst., vol. 20, no. 2, pp. 1134-1143, May 2005.

[3] S. Ganguly, N.C. Sahoo, and D. Das, "Recent advances on power distribution system planning: a state-of-the-art survey," Energy Syst., vol. 4, no. 2, pp. 165-193, Jun. 2013.
[4] C.L.T. Borges and V.F. Martins, "Multistage expansion planning for active distribution networks under demand and distributed generation uncertainties," Int. J. Electr. Power Energy Syst., vol. 36, no. 1, pp. 107116, Mar. 2012.

[5] M.E. Samper and A. Vargas, "Investment decisions in distribution networks under uncertainty with distributed generation-Part I: Model formulation," IEEE Trans. Power Syst., vol. 28, no. 3, pp. 2331-2340, Aug. 2013.

[6] G. Muñoz-Delgado, J. Contreras and J.M. Arroyo, "Multistage generation and network expansion planning in distribution systems considering uncertainty and reliability", IEEE Trans. Power Syst., vol. 31, no. 5, pp. 3715-3728, Sep. 2016.

[7] M. Asensio, P. Meneses de Quevedo, G. Muñoz-Delgado and J. Contreras, "Joint distribution network and renewable energy expansion planning considering demand response and energy storage-Part I: Stochastic programming model," IEEE Trans. Smart Grids, in press. [Online].

http://ieeexplore.ieee.org/stamp/stamp.jsp?arnumber=7462292

[8] S. Wogrin, D. Galbally and J. Reneses, "Optimizing storage operations in medium-and long-term power system models," IEEE Trans. Power Syst., vol. 31, pp. 3129-3138, Jul. 2016.

[9] M. Sedghi, A. Ahmadian and M. Aliakbar, "Optimal storage planning in active distribution network considering uncertainty of wind power distributed generation," IEEE Trans. Power Syst., vol. 31, pp. 304-316, Jan. 2016.

[10]X. Shen, M. Shahidehpour, Y. Han, S. Zhu and J. Zheng, "Expansion planning of active distribution networks with centralized and distributed energy storage systems," IEEE Trans. Sustain. Energy, in press. [Online]. Available: http://ieeexplore.ieee.org/stamp/stamp.jsp?arnumber=7501863.

[11]H. Akhavan and H. Mohsenian, "Energy storage planning in active distribution grids: A chance-constrained optimization with nonparametric probability functions," IEEE Trans. Smart Grids, in press. [Online]. Available: http://ieeexplore.ieee.org/stamp/stamp.jsp?arnumber=7556322.

[12]A. Ahmadian, M. Sedghi and M. Aliakbar, "Fuzzy load modeling of plugin electric vehicles for optimal storage and DG planning in active distribution network," IEEE Trans. Vehicular Technology, in press. [Online]. Available: http://ieeexplore.ieee.org/stamp/stamp.jsp?arnumber=7565635.

[13]Z. Liu, F. Wen and G. Ledwich, "Optimal planning of EVCS in distribution systems," IEEE Trans. Power Deliv., vol. 28, pp. 102-110, no. 1, Jan. 2013.

[14]B. Liu, X. Huang, J. Li, X. Qian and J. Cheng, "Multi-objective planning of distribution network containing distributed generation and electric vehicle charging stations," Dianwang Jishu/Power System Technology, vol. 39 , no. 2 , pp. $450-456$, Oct. 2014.

[15] W. Yao, J. Zhao, F. Wen, Z. Dong, Y. Xue, Y. Xu and K. Meng, "A multi-objective collaborative planning strategy for integrated power distribution and electric vehicle charging systems," IEEE Trans. Power Syst., vol. 29, no. 4, pp. 1811-1821, Jul. 2014.

[16] Y. Weifeng, C.Y. Chung, W. Fushuan, Q. Mingwen and X. Yusheng, "Scenario-based comprehensive expansion planning for distribution systems considering integration of plug-in electric vehicles," IEEE Trans. Power Syst., vol. 31, no. 1, pp. 317-328, Jan. 2016.

[17] National Household Travel Survey (NHTS) data, [Online]. Available: http://nhts.ornl.gov/2009/pub/stt.pdf, 2009.

[18] MATLAB, The Mathworks, Inc., http://www.matlab.com

[19]J. Wu, Advances in K-means Clustering: A Data Mining Thinking. Springer, 2012.

[20]L. Baringo and A.J. Conejo, "Correlated wind-power production and electric load scenarios for investment decisions," Applied Energy, vol. 101, pp. 475-482, Jan. 2013.

[21]D. Arthur and S. Vassilvitskii, "K-means++: the advantages of careful seeding", [Online]. Available: http://ilpubs.stanford.edu:8090/778/1/2006-13.pdf

[22]L. Blank and A. Tarquin, Engineering Economy. New York, NY, USA: McGraw-Hill, 2012.

[23]M. Lavorato, J.F. Franco, M.J. Rider, and R. Romero, "Imposing radiality constraints in distribution system optimization problems," IEEE Trans. Power Syst., vol. 27, no. 1, pp. 172-180, Feb. 2012.

[24] P. Meneses de Quevedo, G. Muñoz-Delgado and J. Contreras, "Joint expansion planning of distribution networks and distributed energy resources including renewable energy, storage and electric vehicles, Data for the case studies, [Online]. Available 
https://drive.google.com/file/d/0Byc0CEUod8ebTEp3RjVRbmZRVFk/vi ew

[25]GAMS Development Corporation, 2015 [Online]. Available: http://www.gams.com 